\title{
"RETRATANDO DIÁLOGOS DE IDENTIDADES" ARTICULACIÓN EDUCATIVA ENTRE LA LICENCIATURA EN PSICOPEDAGOGÍA (UPC), LA ESCUELA ESPECIAL DRA. ANA CAROLINA MOSCA Y EL MUSEO DE ANTROPOLOGÍA (FFYH-UNC)
}

\author{
"PORTRAYING DIALOGUES OF IDENTITIES" \\ EDUCATIONAL ARTICULATION BETWEEN THE BACHELOR'S DEGREE IN \\ PSYCHOPEDAGOGY (UPC), THE DRA. ANA CAROLINA MOSCA SPECIAL \\ SCHOOL AND THE MUSEUM OF ANTHROPOLOGY (FFYH-UNC)
}

\section{Mariela Eleonora Zabala ${ }^{a^{*}}$ y Mariana Beatriz Dalmasso ${ }^{b}$}

Fechas de recepción y aceptación: 30 de octubre de 2020 y 11 de abril de 2021

DOI: https://doi.org/10.46583/edetania_2021.59.754

Resumen: Proponemos dar a conocer una experiencia de pedagogía museística y reflexionar sobre las experiencias de trabajo interdisciplinario e interinstitucional desarrolladas en el proyecto artístico "Retratando diálogos de identidades", desde el diálogo de saberes y la extensión universitaria. Este fue creado colectivamente entre profesionales pertenecientes a dos espacios de formación superior y universitaria de la ciudad de Córdoba (Argentina), y de la Escuela Especial Dra. Ana Carolina Mosca. Participaron las/os practicantes de la carrera de Psicopedagogía de la Universidad Provincial de Córdoba y docentes, no docentes, estudiantes, egresados/as y personal de seguridad del Museo de Antropología de la Universidad Nacional de Córdoba. Creamos y coordinamos el proyecto psicopedagogas, artistas plásticas y antropólogas. Este se desarrolló entre el año 2018 y 2019.

El objetivo fue habitar el museo con una propuesta de intervención artística para jóvenes con necesidades educativas derivadas de la discapacidad y acompañados por estudiantes del último año de la licenciatura de Psicopedagogía. Para estos últimos el proyecto también tuvo

\footnotetext{
${ }^{a}$ Departamento de Antropología. Facultad de Filosofía y Humanidades. Universidad Nacional de Córdoba.

${ }^{\mathrm{b}}$ Directora de la Escuela Especial El Amanecer. APADIM. Las Varillas. Docente del seminario Práctica Docente I, en el profesorado de Educación Especial en el Instituto María Inmaculada.

${ }^{*}$ Correspondencia: Universidad Nacional de Córdoba. Pabellón Residencial. Ciudad Universitaria Córdoba. Argentina.

E-mail: marielazabala@ffyh.unc.edu.ar
} 
como finalidad problematizar la institución museo como un espacio potente para enseñar y aprender con personas con discapacidad intelectual.

Las estrategias didácticas seleccionadas se enmarcaron en los fundamentos del aprendizaje situado, que postula una propuesta de enseñanza entre los procesos escolares y la realidad social, es decir el entorno en donde se produce el conocimiento.

A través de esta escritura queremos documentar lo no documentado de las experiencias educativas en un museo universitario y a partir de esa descripción analizar lo vivido.

Palabras claves: museo, escuela especial, formación profesional, diálogo ¿solo o de saberes?, derechos humanos.

Abstract: We present an experience of museum pedagogy and reflect on the experiences of interdisciplinary and inter-institutional work developed in the artistic project "Portraying dialogues of identities", from the dialogue of knowledge and university extension. It was created collectively between professionals belonging to two spaces of university and higher education in the city of Córdoba (Argentina), and the Dra. Ana Carolina Mosca Special School. The interns of the Psychopedagogy degree of the Provincial University of Córdoba and teachers, non-teachers, students, graduates and security personnel from the Museum of Anthropology of the National University of Córdoba participated. The project is created and coordinated by us psychopedagogues, plastic artists and anthropologists. It was developed between 2018 and 2019.

The objective was to inhabit the museum with a proposal of artistic intervention for young people with educational needs related to disabilities and accompanied by students of the last year of the bachelor's degree in Psychopedagogy. For the latter, the project also aimed to problematize the museum institution as a powerful space for teaching and learning with people with intellectual disabilities.

The selected didactic strategies were framed in the foundations of situated learning, which postulates a teaching proposal between school processes and social reality, that is, the context where knowledge is produced.

Through this writing we want to document the undocumented of educational experiences in a university museum and to analyze what was lived.

Keywords: museum, special school, vocational training, dialogue, alone or of knowledge?, human rights.

\section{INTRODUCCIÓN}

Nos proponemos reflexionar sobre las experiencias de trabajo interdisciplinario e interinstitucional desarrolladas en el proyecto artístico "Retratando diálogos de identidades", desde el diálogo de saberes, la extensión universitaria y el aprendizaje situado. Este fue creado colectivamente entre profesionales 
pertenecientes a dos espacios de formación superior y universitaria de la ciudad de Córdoba (Argentina), y de la Escuela Especial Dra. Ana Carolina Mosca. Participaron las/os practicantes de la carrera de Psicopedagogía de la Facultad de Educación y Salud de la Universidad Provincial de Córdoba (UPC) y docentes, no docentes, estudiantes, egresados/as y personal de seguridad del Museo de Antropología de la Facultad de Filosofía y Humanidades de la Universidad Nacional de Córdoba (MA). Creamos y coordinamos el proyecto psicopedagogas, artistas plásticas y antropólogas. Este se desarrolló entre el año 2018 y 2019.

El objetivo fue habitar el Museo con una propuesta de intervención artística para jóvenes de educación de especial acompañados por los/las practicantes. Para estos últimos, el proyecto también tuvo como finalidad problematizar a la institución museo como un espacio potente para enseñar y aprender con personas con discapacidad intelectual.

Recuperando los registros que pedagógicamente documentaron la experiencia, tanto en el museo como en las aulas de la universidad y de la escuela, queremos socializar la experiencia y los saberes creados colectivamente, así como los interrogantes y reflexiones que surgieron en el desarrollo del proyecto. Partimos de la premisa de entender la educación, la cultura y la salud como derechos humanos indisociables en cuanto a su acceso y ejercicio. Consideramos que compartir la experiencia vivida desde las y los actores sociales involucrados es un modo de conocer el mundo social que habitamos, y pensar nuevos vínculos entre escuela, universidades y museo.

\section{Trabajo interinstitucional: el Museo de Antropología,} La Universidad Provincial y la escuela de modalidad ESPECIAL DRA. CAROLINA MOSCA

El proyecto surgió en el MA cuando nos reunimos docentes de ambas universidades que veníamos participando en otro proyecto extensionista llamado "Necesito observar una visita guiada: una propuesta de articulación entre el Instituto Superior de Formación Docente y el Museo de Antropología" (Zabala y García Armesto, 2016; Zabala, Zabalza y García Armesto, 2017). En este marco, la docente y psicopedagoga Mariana Dalmasso vio en la institución 
museística un espacio potencial para las/os estudiantes, tanto de la escuela como de la licenciatura, que llevarían a cabo una práctica educativa diferente, que aportara al diálogo sobre la identidad desde el arte a través de conversatorios sobre las biografías de los trabajadores del museo. Cabe señalar que el vínculo entre el museo y la escuela existe desde el año 2002, ya que una vez al año el museo era visitado por las y los estudiantes. Ahora nos animamos a repensar la "visita" al museo y el vínculo entre museo y escuela. Como una de las características del aprendizaje situado es el trabajo fuera del aula, de la escuela, consideramos necesario describir las tres instituciones involucradas.

El MA es una institución de carácter técnico-científico, con objetivos educativos y culturales, tendientes a preservar y revalorizar el patrimonio cultural de la región. En él se desarrollan proyectos de formación docente, investigación y extensión, que buscan dar respuesta a distintas demandas de comunidades extrauniversitarias. Tiene como misión educar sobre la cultura de las sociedades indígenas y locales, pasadas y contemporáneas, como una manera de fomentar el respeto hacia otros modos de vida y de crear actitudes de preservación del patrimonio cultural en la sociedad (Bonnin, 2007; Exp. 12/02/24545 Ord. n. ${ }^{\circ}$ 01/02). Desde su reapertura en el año 2002, entiende a la extensión como parte de su misión y tiene la "voluntad deliberada" de vincularse con la sociedad a través de la gestación de proyectos de inclusión social. Entiende esta función universitaria como la creación y el derecho a determinados espacios y bienes culturales, y la generación de nuevos conocimientos en el encuentro con otros/ as ciudadanos/as. Así se produce un diálogo de saberes multidireccionales que lo enriquecen.

La escuela de modalidad especial pertenece a la educación pública estatal y cuenta con los niveles primario y secundario. Está ubicada en la ciudad de Córdoba, a cuadras del centro histórico y del Museo de Antropología, distancia que puede ser recorrida caminando. En el marco de la Ley Nacional de Educación n. ${ }^{\circ}$ 26.206, se rige por el principio de inclusión, asumiendo el desafío de elaborar propuestas educativas que promuevan prácticas inclusivas en escenarios socioculturales, más allá de las paredes escolares. Adherimos a la concepción de la discapacidad desde el modelo social que afirma que "La discapacidad es una situación heterogénea que envuelve la interacción de una persona en sus dimensiones física o psíquica y los componentes de la sociedad en la que se desarrolla y vive" (Muñoz Padilla, 2010, p. 384). La población 
estudiantil es heterogénea respecto a las etiologías que definen las estrategias cognitivas que poseen los y las jóvenes. Están asociadas a factores genéticos, adquiridos, ambientales y socioculturales. Ingresan a la institución, luego de un proceso de evaluación interdisciplinaria de las trayectorias escolares, realizadas por nuestro sistema educativo. Provienen de distintos barrios de la ciudad de Córdoba. En su mayoría de hogares con ingresos económicos mínimos, algunos en situación de analfabetismo, luego de haber transitado (con repitencias y hasta deserción) los primeros años de escolaridad en las escuelas de nivel primario (común).

El trabajo en conjunto con la Universidad Provincial es más nuevo porque la institución fue creada recientemente en el año 2007, y tiene el encanto de ofrecer la formación en Psicopedagogía, que no tiene la Universidad Nacional pero que es muy importante para pensar prácticas de inclusión socioeducativa en los museos. Su plan de estudio comprende cinco años. La psicopedagogía tiene como objetivo en su abordaje al sujeto en situación de aprendizaje (Giannone y Gómez, 2011), en la diversidad de situaciones y en los avatares del aprendizaje como proceso, en una trama relacional en un contexto definido. La práctica del último año demanda del futuro psicopedagogo una posición reflexiva y creativa para evaluar una situación y definir propuestas de intervención.

Si bien el vínculo entre escuela y museo no es nuevo, ya que data de la emergencia de los museos en Argentina, a finales del siglo XIX, estos museos han sido instituciones estatales pensadas para crear una identidad nacional colectiva, enseñar sobre un pasado en común y producir conocimientos legitimados; en este caso se buscaba una intervención por parte de estudiantes de nivel medio y practicantes universitarios en el museo para recrear sus identidades. Para los primeros fue clave su condición de "discapacidad" y para los/as otros/as, su campo profesional. Estos lazos entre escuelas y museos continúan, pero resemantizados, y esto es lo que queremos poner en tensión a partir de la reflexión de esta experiencia vivida.

\subsection{El proyecto}

La actividad conjunta que nos convocaba era la producción de un "telón de identidad institucional" para el MA. Esta tuvo como antecedente la 
experiencia de realización, por el mismo grupo de alumnos/as y docentes, del "telón mosquetero". Este fue realizado en la escuela durante los ciclos lectivos 2016-2017 y tuvo como objetivo "describir el proceso inter e intrasubjetivo, en el diseño y elaboración de un símbolo de identidad institucional" (Andrada et al., 2018, p. 48).

Entendimos que no hay arte sin personas, pero quizá tampoco personas sin arte. Por él, el mundo se hace más inteligible y accesible, más familiar, más accesible, más aprehensible. Es el medio de un perpetuo intercambio con el entorno inmediato, una especie de respiración del alma, bastante parecida a la física, sin la que no puede pasar nuestro cuerpo (Huyghe, citado en Alderoqui et. al, 1995, p. 5).

La expresión plástica elegida fue la de retrato, es decir la pintura o efigie de una persona donde predomina el rostro y su expresión. El retrato se propone como imagen de identidad. La definición de retrato también se aparta de la idea de modelo fiel para acercarse a la de conjunto de signos donde cada uno, sea este el/la pintor/a o el/la espectador/a, reconstruye a su gusto la imagen de una persona apenas determinada, que ha transcurrido en el lapso de tiempo que puede ser alcanzado todavía por nuestros recuerdos vividos. El retrato constituyó el género que escenificó la voluntad de concretar la identidad del hombre en el tiempo (Priego, 1989, p. 7). Si bien el avance tecnológico permite nuevas formas para la representación individual y colectiva, se recurre al retrato pintado para habilitar el intercambio de saberes entre actores de diferentes instituciones.

Los intereses que movilizaron la realización del proyecto fue la de generar nuevas praxis en futuros/as psicopedagogos/as que hasta el momento identificaban sus prácticas profesionales en centros educativos, de salud y/o consultorios privados. Por otra parte, era necesario extender el proyecto de artes visuales en otros espacios más allá de la escuela, con jóvenes que quieren mostrar sus capacidades para el trabajo artístico, de manera que se otorga un nuevo significado a los preconceptos y prejuicios sociales respecto a las personas con "discapacidad". El MA, por su parte, estaba comenzando una nueva gestión que buscaba pensar la identidad institucional y reconocer la diversidad constitutiva de todo el equipo de trabajo.

En el caso del MA participaron sus miembros, pertenecientes a sus cuatro claustros universitarios (docentes, no docentes, egresados/as y alumnos/as) así 
como los/as investigadores/as y el personal de seguridad. Por parte de la escuela, se involucraron los/as docentes de artes visuales, auxiliares y miembros del gabinete psicopedagógico y los/as estudiantes de tercero y cuarto año del nivel secundario. El grupo estuvo conformado por mujeres y varones, sumando un total de 15 jóvenes. Las modalidades de aprendizaje son diversas: cognitivamente se desplegaron estrategias que derivan de un pensamiento intuitivo ${ }^{1}$ y de las operaciones concretas, el acceso al conocimiento se produjo a través de recursos visuales, auditivos y kinestésicos, la expresión escrita comprende la alfabetización plena, así como la escritura a cargo de otro/a (maestro/a o compañero/a). Como factor común entre los/las jóvenes, necesitan la generación de experiencias de aprendizajes significativas, que permitan la integración de los conocimientos en el abordaje de situaciones reales. Aprender con sentido.

Los/las practicantes de la licenciatura de psicopedagogía estaban cursando quinto año y el espacio curricular denominado Práctica V cuyo eje son los proyectos de abordajes psicopedagógicos en diferentes ámbitos. Estos conformaban un grupo de 5 integrantes acompañados por su docente, en su mayoría mujeres. El espacio de la práctica psicopedagógica demanda a los/las practicantes la elaboración de estrategias de intervención en un ámbito definido mientras se va elaborando, a su vez, la identidad profesional.

\subsection{Fundamentos pedagógicos}

Teniendo en cuenta la propuesta de intervención en el MA y el trabajo interinstitucional e interdisciplinar, elegimos generar el proyecto desde los postulados del aprendizaje situado. Es decir, lo aprendido se explica solo a partir de las prácticas sociales en la vida cotidiana, la vida diaria. Lo "situado" del aprendizaje es el resultado de la relación entre quienes aprenden y el entorno sociocultural en el que ejercen la acción y/o actividad. Uno de los objetivos es el desarrollo de competencias más que la adquisición de conocimientos

${ }^{1}$ El pensamiento está centrado en un solo aspecto del objeto que se va a conocer. Sus ideas se basan en las percepciones. En el estadio de las operaciones concretas, el pensamiento se caracteriza por el desarrollo de la reversibilidad, lo que permite conservar la naturaleza de los objetos y sus diferentes características. 
(Sagástegui, 2004). El conocimiento "es parte y producto de la actividad, del contexto y la cultura en que se desarrolla y utiliza" (Díaz Barriga, 2003, p. 2), lo que incentiva el trabajo en equipo y cooperativo. Tres elementos son claves en este proceso de enseñanza y aprendizaje: la pertenencia, la participación y la práctica. El aprendizaje, entonces, se define como una experiencia social donde el conocimiento que se genera es contextual, y los estudiantes se apropian de herramientas que amplían sus habilidades y saberes. Para Wassermann, los proyectos deben incluir actividades que requieran que los/las estudiantes investiguen, construyan y analicen información que coincida con los objetivos específicos de la tarea (1994, p. 160). La narrativa es la herramienta protagonista en la que confluyen “(...) información y datos: psicológicos, sociológicos, científicos, antropológicos, históricos y de observación, además de material técnico" (Wassermann, 1994, p. 5). El contenido de la narrativa es interpelado por las preguntas críticas, aquellas que permitan indagar conceptos centrales y situaciones problemáticas que se presenten durante el proceso de retratar diálogos de identidades. El objetivo es la elaboración de conocimiento reflexionado en el acontecer del proyecto en sus distintas etapas y en grupos de trabajo. En estos últimos, tienen lugar las interacciones en las que circulan las preguntas críticas entre estudiantes, practicantes, retratados y docentes. La tarea docente consiste en guiar la discusión para el análisis y apropiación de significados. Entonces se producen las modificaciones en las formas de comprensión y participación de los sujetos en una actividad conjunta. En este proceso, los/las docentes y guías del museo fueron propiciadores, acompañantes y facilitadores.

\subsection{Las secuencias didácticas}

La secuenciación fue multisituada para los/as estudiantes, ya que tuvo lugar en la escuela y en el museo; mientras que para los/as practicantes se agregó un espacio más, las aulas de su facultad. Asimismo, hubo momentos diferenciales de trabajo por institución, así como momentos compartidos. A través de estas secuenciaciones de aprendizajes de habilidades, destrezas y conceptos buscamos vincular el mundo escolar, el mundo profesional con el MA. 


\subsubsection{Para los estudiantes del Mosca}

Se realizó la presentación del proyecto y de los/las practicantes en la escuela. Se preparó la documentación para salir a visitar el museo.

Se organizó la visita al museo para conocer sus instalaciones, más allá de sus salas de exhibición y sus trabajadores/as. Como instancia de previsita, las/os estudiantes de la escuela tuvieron sus instancias preparatorias, a cargo de las/os practicantes en psicopedagogía. Para ello diseñaron y desarrollaron dos talleres. En estos abordaron "las representaciones sobre un museo y en particular del Museo de Antropología"; y en los siguientes: "qué es una biografía y el vínculo con la identidad". Así fue como recorrimos los laboratorios de investigación, la biblioteca, las oficinas, la recepción y las distintas áreas del museo para que conocieran a la gente que luego iban a retratar. En estas mismas instancias visitamos los baños, la cocina, el ascensor, las escaleras y los lugares posibles donde ellos/as iban a poder retratar.

Luego pasamos al armado de grupos con estudiantes y practicantes para la realización de retratos en el museo. Hicimos el cronograma de jornadas de retratos. En estas se harían los bocetos, se tomarían fotografías y se propondría la actividad de la biblioteca humana. Los retratos fueron libros que se "leyeron" en las conversaciones, mediados por el/la practicante. El/la retratado/a contaba quién era y qué hacía en el museo, y ahí fluía el diálogo sobre el museo y sus tareas y funciones, así como qué era ser antropólogo/a y/o arqueólogo/a. Nos inspiramos en la propuesta realizada en Copenhague en 1993 donde los/ las protagonistas también eran jóvenes y tenía como fin incentivar el diálogo para fomentar el entendimiento a través del intercambio de conocimientos, sentimientos y emociones.

El/la practicante registró el momento del encuentro entre retratados/as y retratadores, por escrito y en imágenes. Con este material realizaron una actividad lúdica que fortaleciera las prácticas de lectura y escritura, con sentido comunicativo, en los jóvenes de la escuela. Los materiales didácticos creados fueron un libro de biografías y un juego de mesa en el que se representa el recorrido del museo con el encuentro de los/as retratados/a y retratadores.

En la escuela se concluyeron los bocetos de 49 trabajadores/as y se pasó al lienzo (50 x $50 \mathrm{~cm}$ cada lienzo). Junto con la docente eligieron la resolución pictórica del fondo y el color del lienzo. Esta fue una actividad grupal. 
Se numeraron las obras en filas y en columnas. Cuando todos los retratos estuvieron listos, se inició el hilvanado, utilizando la estrategia temporal del "alfiler", y por último la costura del telón. Durante estas jornadas en la Escuela participamos algunos miembros del museo así como los practicantes.

Finalmente se hizo un colgado y presentación del telón en la escuela y el MA. A esta instancia última fueron invitados los familiares de los/as estudiantes. Esta presentación pública fue acompañada con proyección de videos que reconstruían el proceso realizado, pintura de retratos en vivo e interpretación de rap a cargo de les estudiantes "del Mosca".

\subsubsection{La secuencia para los/las practicantes}

El tiempo de la práctica psicopedagógica en quinto año se desarrolla en la construcción del conocimiento profesional. Basado en las pautas curriculares, el análisis dialogado se produce entre la situación en la que participan y los saberes elaborados, marcando así el ritmo de la secuencia didáctica. Se conjugan de este modo: situación, participación, reflexión e intervención.

Para materializar la circulación de preguntas críticas y la discusión en los grupos de trabajo se utiliza la propuesta de Erausquin y Basualdo (2010), quienes ofrecen orientaciones para la reflexión en y desde la práctica.

La secuencia didáctica se desarrolla del siguiente modo, durante el período de un ciclo lectivo:

Relato de la situación-problema, expuesta en el artículo “Telón Mosquetero. Un símbolo de identidad institucional”. Constituye la primera aproximación de abordaje de jóvenes, discapacidad y arte. Se destaca el valor del arte como lenguaje emancipador, la descripción del proceso creativo, el quehacer psicopedagógico en la orientación pedagógica dentro de la integración de espacios curriculares, así como la reconstrucción del proceso y la proyección hacia espacios socioculturales. Específicamente la realización del telón de identidad institucional del Museo de Antropología de la Facultad de Filosofía y Humanidades de la Universidad Nacional Córdoba.

Aproximación al campo, presentación en el MA, recorrido por este y reunión en la biblioteca con el objetivo de definir el rol psicopedagógico. Paralelamente se inicia la asistencia regular a la escuela de modalidad en el espacio curricular 
de artes visuales, se realizan entrevistas con docente del área y se establecen vínculos con los/las estudiantes, con quienes realizarán un trabajo colaborativo.

Después se lleva a cabo la práctica reflexiva a partir de la matriz de análisis:

Dimensión situación-problema: de la descripción a la explicación del problema. Los/as practicantes describen las clases observadas en artes visuales: dan cuenta de la interrelación entre consignas y posicionamiento de docente y estudiantes. Surgen los siguientes interrogantes: ¿Cuál es la relación entre arte y discapacidad? ¿Cómo vincular escuela y museo? ¿Cuál es la intervención posible del psicopedagogo? La guía docente en la construcción de un conocimiento situado propone como recorrido de lectura conceptos centrales respecto al arte como actividad experiencial ${ }^{2}$. El concepto de discapacidad desde el paradigma del déficit ${ }^{3} \mathrm{y}$ de la diferencia se articula con el concepto de modalidad de aprendizaje ${ }^{4}$. Respecto a la visita al museo, se realiza la delimitación de la participación de los/as jóvenes estudiantes de modalidad especial y los aprendizajes posibles proyectados.

La descripción del escenario escolar movilizó representaciones de los/las practicantes sobre la escuela especial y sus destinatarios/as. Se reconoce la intención pedagógica de la institución por la representación rehabilitadora de esta; la habitan estudiantes y no pacientes, hay aulas con grupos de trabajo y no consultorios con atención individualizada. La clase de artes visuales consiste en el despliegue de un taller creativo atravesado por los objetivos planificados por el docente. Los/as jóvenes han desarrollado la habilidad del diseño autónomo del retrato, disponiendo el uso pertinente de hojas, lápices, pinceles, telas, acrílicos, libros de referencia, y dan cuenta de modo verbal del proceso que realizan. Se visualiza la diversidad de recursos utilizados que se

${ }^{2}$ Augustowsky, G. (2012). "El arte en la Enseñanza". "El arte como experiencia estética, Dewey, relaciona el arte con lo cotidiano, con el cuerpo, con la actividad y con la Creación. Para Dewey el arte no es algo lejano, esotérico, sino que tiene una función en nuestras vidas. El arte es una actividad experiencial, tanto en su producción como en su recepción" (pp. 28-29).

${ }^{3}$ Aznar A. Castañon González D. (2008) ¿Son o se hacen? "En el paradigma del déficit se compara cuantitativamente a los objetos-en este caso las personas con limitaciones funcionales, con respecto a un patrón o modelo sancionado como normal. Mientras que desde el paradigma de la diferencia, esta última es una característica que distingue uno u otro, uno de otro". (p. 22-23)

${ }^{4}$ Fernández A. (2003) Los idiomas del Aprendiente “(...) es el molde o esquema de operar que se va a ir utilizando en las diferentes situaciones de aprendizaje" (p. 93). 
articulan con la diversidad de modos de acceder al objeto de conocimiento, porque hay diversidad de modalidad de aprendizajes.

Acuerdos conceptuales: nos posicionamos en el arte como lenguaje subjetivante, la discapacidad se entiende desde el paradigma de la diferencia y la visibilidad de las modalidades de aprendizajes existentes como expresión de las condiciones de aprendizajes de los/as estudiantes de la escuela especial. Se destaca la importancia de leer a los/as jóvenes en términos de sus modalidades de aprendizajes, ya que los habilita para aprender.

En la interacción con los/as representantes del museo se establece la necesidad de intervenir en la vinculación de estudiante con el museo en un abordaje colaborativo en el registro de las biografías.

Dimensión intervención profesional: la decisión de intervención es elaborada de manera colaborativa desde los acuerdos que se derivan de las respuestas a las preguntas críticas. Se indican acciones articuladas entre sí y la distribución de tareas, en relación con los objetivos enunciados. Se dividen en tres momentos:

- Antes de retratar: se realiza la visita de reconocimiento al museo junto a los/as estudiantes, se registra de modo escrito y audiovisual cada espacio junto al relato de quienes lo habitan. Se diseñan y desarrollan dos talleres para contextualizar la visita al museo y el proyecto: "las representaciones sobre un museo y en particular del Museo de Antropología” y "qué es una biografía y el vínculo con la identidad".

- Durante la asistencia al MA, para realizar los retratos: guía y registro de las biografías narradas por los/as retratantes, a través de la estrategia de biblioteca humana.

- Después de la realización de los retratos: sistematización de las narrativas biográficas, reconstrucción de los espacios del museo desde los significados otorgados por los trabajadores del museo, acompañamiento en el reconocimiento de cada retratado de su retrato.

Dimensión herramientas: se disponen herramientas en relación con dos aristas del problema: el vínculo escuela-museo y los talleres con eje en el intercambio dialogado, modalidad de aprendizaje-discapacidad (paradigma de la diferencia), elaboración de libro (relato escrito) y dispositivo lúdico. Ambas asociadas al rol profesional psicopedagógico. 
Dimensión resultados: se establecen resultados en relación con las condiciones pedagógicas que se planificaron. Esto va más allá de la materialización de objetos como evidencias de aprendizajes: en el libro de biografías y el dispositivo lúdico se reconocen como construcción de conocimiento los siguientes postulados:

- Relación arte y discapacidad: esencialmente comprender la discapacidad desde el paradigma de la diferencia permite visibilizar a los/as estudiantes como sujetos de aprendizaje con diversidad de estilos, para no permanecer en diagnósticos rotulantes. A través del arte visual es posible movilizar y conocer la diversidad de modalidades de acceso al objeto a partir de los recursos didácticos utilizados.

- Relación escuela y museo: se define desde los/as sujetos que la habitan. Sujetos historizados en los que ambas instituciones pueden promover estrategias que disminuyan las barreras de participación en espacios socioculturales.

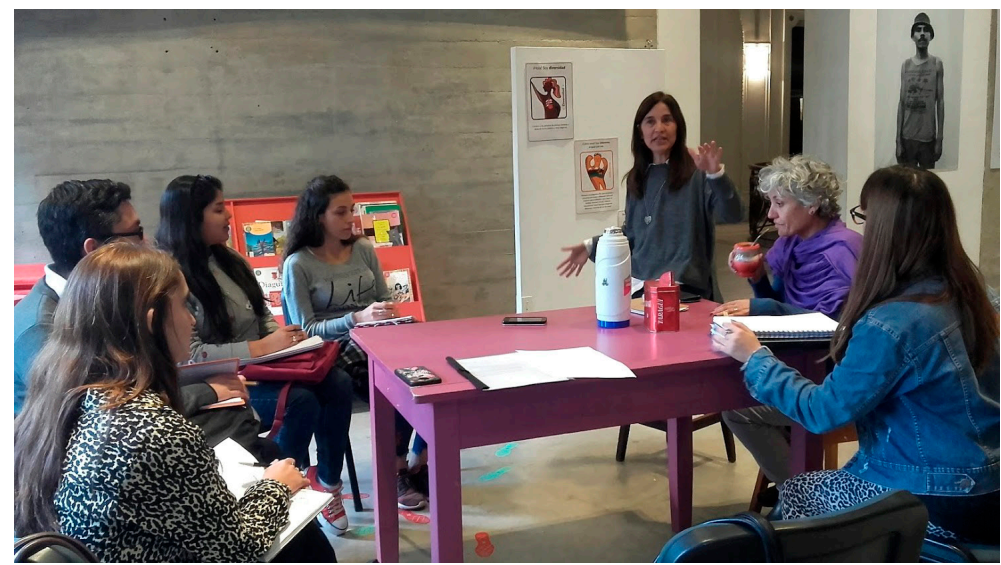

Fotografía tomada por Mariana Dalmasso. Muestra los momentos de reunión en el Museo de Antropología. 

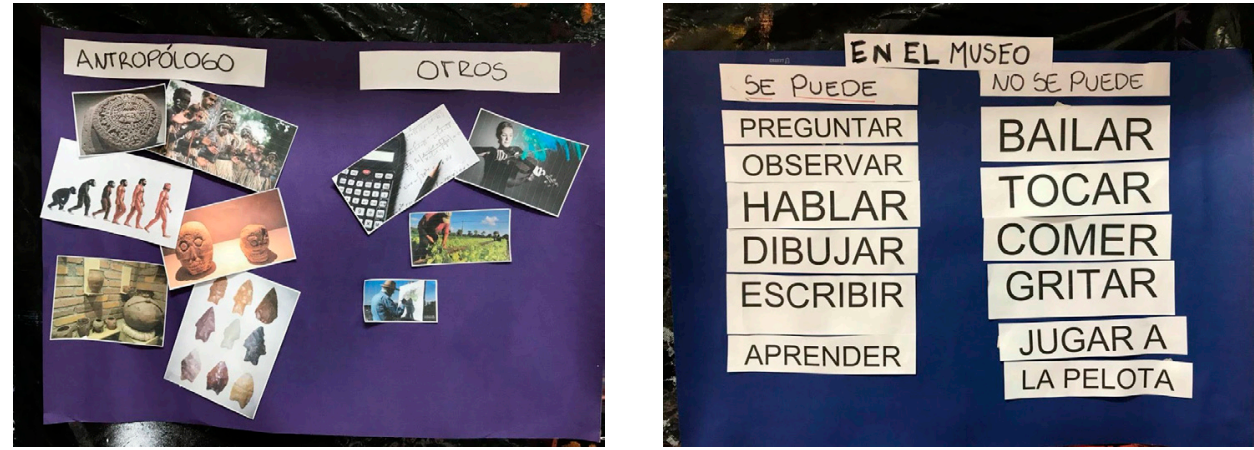

Fotografía tomada por Mariana Dalmasso. Muestra las producciones de los talleres en la escuela propuestos por los estudiantes de la UPC.
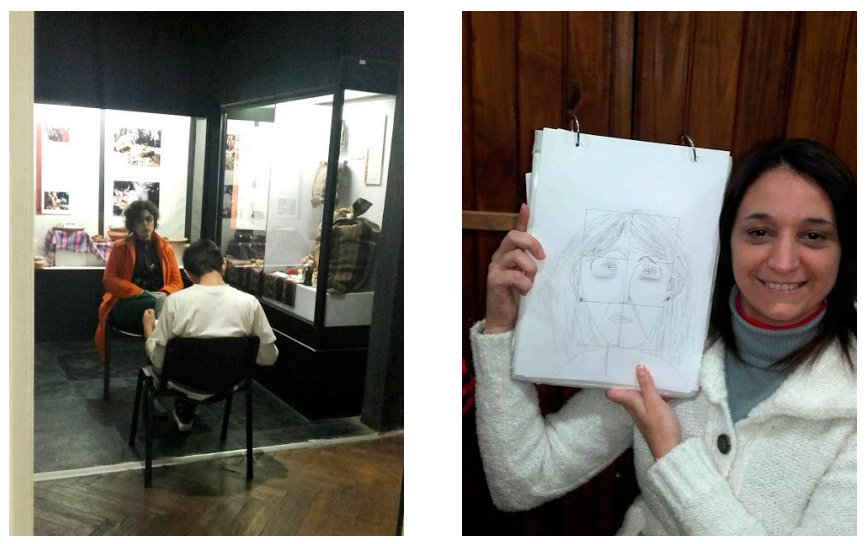

Fotografía tomada por Mariana Dalmasso. Muestra el momento de realización de los bocetos en el Museo de Antropología. 


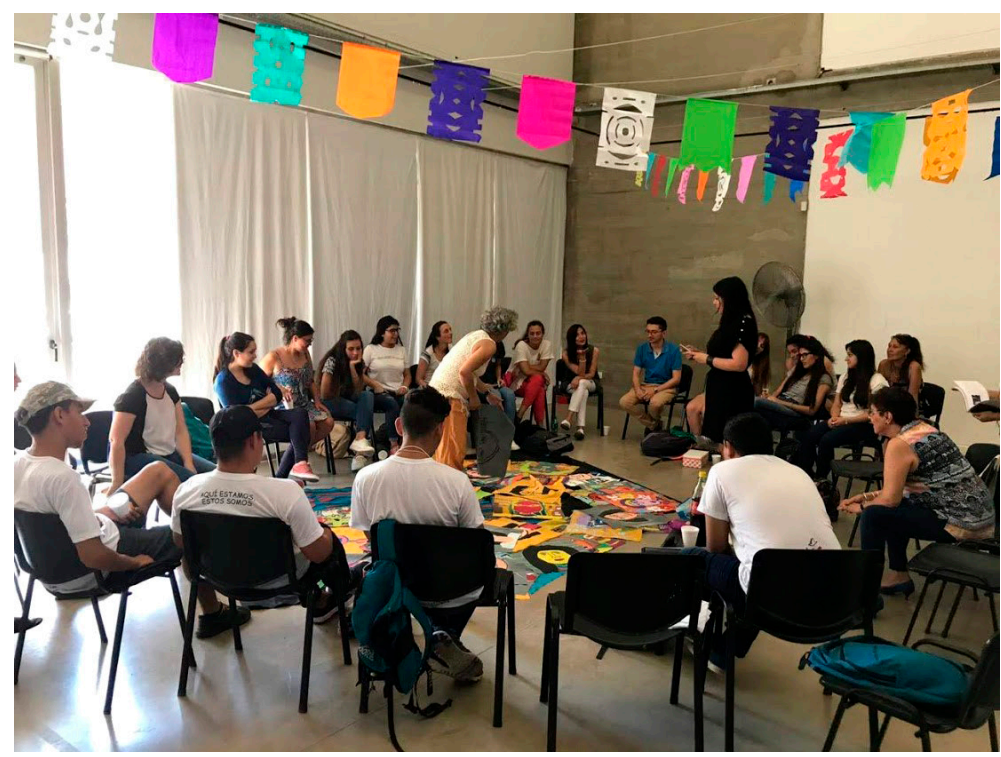

Fotografía tomada por Mariana Dalmasso. Muestra el momento de presentación e inauguración del telón en el Museo de Antropología.

\section{REFLEXIÓN DE LA PRÁCTICA}

Recuperamos palabras de Edelstein, quien define la enseñanza como una “... actividad intencional, que pone en juego explícita o implícitamente distintas racionalidades, el interés es justamente poner en situación de análisis la intencionalidad, la racionalidad que subyace en cada caso. En suma, los mecanismos más sutiles que permiten develar el sentido y orientación de esta práctica" (2000, p. 4). Dicho esto, consideramos significativo recuperar la voz de los/as practicantes como una búsqueda sistemática de claves que permitan una mayor comprensión de la práctica docente en un museo.

La valoración de la experiencia en palabras de las/los practicantes: "los sujetos pudieron posicionarse como autores, dejando inscripta una huella en la sociedad, desplegaron sus potencialidades, interactuaron con otros, se sintieron protagonistas del proyecto"; "...consideramos que dicha experiencia posibilita la subjetivación, apartando la connotación del rótulo de discapacitado 
que muchas veces viene impuesto por la sociedad". "No alcanza solamente con decir que se valoran las diversidades, sino que se deben poner en juego espacios de participación y desenvolvimiento de las diferencias para que los encuentros sean ricos". Ellos vivenciaron "acompañando a los/as adolescentes a sentirse autores/as de sus propios trabajos, a poder tomar la palabra frente a quienes retratan y generar confianza en sí mismos".

La psicopedagogía intenta desnaturalizar los discursos sociales en torno a la discapacidad para dar lugar a que "otras cosas" ocurran, romper con las representaciones sociales sobre la discapacidad y poder promover en el sujeto su autonomía con el fin de incluirse dentro de la sociedad, procurando que estas sean participantes activos/as de su comunidad desde sus talentos. La experiencia posibilitó el quehacer psicopedagógico, desde un rol vinculante tramitando la relación con el MA y sus trabajadores/as y vivenciando un posicionamiento subjetivo de autoría, en los/las jóvenes de la escuela especial.

La actividad conjunta que nos convocó fue la realización de un telón de identidad institucional en el que la relación parecía sostenerse en la tela, para plasmar la identidad de otros, pero el museo nos alojó y las narrativas singulares se transformaron en colectivas y dio lugar a la construcción de un nuevo significado identitario ahora: "El museo somos todos".

El desarrollo del proyecto visibiliza que el principio de inclusión puede trascender de un ideario institucional a prácticas subjetivantes, que conlleva un abordaje ético interdisciplinario e interinstitucional en la mirada de los/as sujetos que habitan en ellos para habilitar la participación plena.

\section{Conclusiones}

En este proyecto no es posible distinguir "los/las destinatarios/as", ya que todos fuimos productores/as y destinatarios/as como lo postula el aprendizaje situado. Todos/as estuvimos involucrados con tareas y roles diferentes, pero no basándonos en desigualdades cognitivas, sino en habilidades, destrezas y responsabilidades. No hubo transferencia de conocimientos, sino una circulación de pensamientos y conocimientos que se pusieron en diálogo. Hubo lógicas institucionales que se pusieron en diálogo, hubo una agenda de trabajo construida colectivamente con días, horarios y actividades. Fue un proyecto que 
implicó acción y reflexión sobre las necesidades que iban surgiendo, desde las compras de los materiales hasta qué imaginario social había de la institución museo, y qué representación había acerca de la discapacidad.

Con respecto a la Universidad Nacional tomamos una actitud reflexiva para que no ocupe el lugar históricamente construido del monopolio del saber, de transmisora de conocimientos sino de hacer un trabajo de extensión donde seamos un actor más. Hubo una participación en forma de igualdad, respeto y reciprocidad, entendiendo dialógicamente nuestras autoidentificaciones, y las formas de construir y practicar el conocimiento (Mendoza, 2016, p. 34).

Nos propusimos construir y sostener una organización comunitaria de aprendizaje colectivo, donde pusimos en juego habilidades diversas a través del trabajo cooperativo. Vivenciamos una experiencia de aprendizaje intercultural a partir de la articulación de la cultura escolar y extraescolar de una institución de nivel medio y superior, y la cultura de un museo universitario. Allí fuimos capaces de transitar la incertidumbre y la convertimos en posibilidad de aprendizaje.

"Retratando diálogo de identidades" fue un proyecto que generó situaciones potentes de aprendizaje para:

- La formación del rol psicopedagógico, que posibilitó abordar las modalidades de aprendizajes en la discapacidad, en la relación educaciónmuseo, mediada por el arte visual y el diálogo, problematizando la institución museo como un espacio potente para enseñar, aprender y gozar. Siguiendo a Chagas (2007), entendemos los museos como espacios que generan respuestas singulares a problemas singulares de la sociedad con compromiso político y poético. En nuestro caso el compromiso político fue con la inclusión social en los museos y la poética fue la creación artística de retratos.

- Los/as jóvenes con discapacidad, que trascendieron de la técnica del retrato a la autoría de un telón de identidad institucional como parte del patrimonio del MA.

- Para los/las trabajadores/as del museo, la posibilidad de crear un proyecto desde la extensión universitaria, es decir un modo de relacionarnos con la sociedad de la cual somos parte y con la cual coconstruimos conjunta, solidaria y comprometidamente los conocimientos para la resolución de problemáticas socialmente definidas (Pacheco, S/D). Este proyecto fue 
posible porque llevábamos tiempo trabajando conjuntamente en distintas propuestas del museo, pero en esta cambió, y el museo pasó a ser el que recibe la demanda, no el que ofrecía la propuesta educativa. Fue la posibilidad de ampliar e incorporar una problemática de la universidad provincial y de la escuela especial a la agenda del museo, fortaleciendo el trabajo con comunidades en el territorio.

De este modo el museo dejaba de ser un espacio a donde se va solo de "visita" para ser un lugar de "clases", "un taller de dibujo", "un lugar de encuentro". Así se pasa de ser oferente a un trabajo colaborativo con otras instituciones educativas. En palabras de Navajas Corral (2008), la institución museo está pensando en el territorio y la comunidad que lo habita, y el patrimonio nunca acabado que elige, interpreta y crea mancomunadamente. De este modo se vivencia un espacio abierto, interactivo y multidisciplinar.

Inspiradas en Scheiner (2017), consideramos que el museo con este proyecto se convirtió en un lugar que pudo movilizar sentimientos y emociones siendo habitado por una propuesta creativa-artística, que permitió a las/los participantes transformarse en pro del bien común y el bienestar social. El museo se volvió un atelier.

\section{BiBLIOGRAFÍA}

Alderoqui, H. et al. (1995). Documento de Trabajo n. $^{\circ}$ 1. Actualización Curricular. Buenos Aires: Dirección de Currícula de la Municipalidad de la ciudad de Buenos Aires.

Alderoqui, S. (2010). Política y poética educativa en museos. Entre los visitantes y los objetos. Museos argentinos/investigaciones 02. Recuperado de: https://cutt.ly/rbwnHut

Andrada, G. et al. (2018). Telón Mosquetero: un símbolo de identidad institucional. Novedades Educativas 236, 48-49.

Augustowsky, G. (2012). El arte en la Enseñanza. Buenos Aires: Paidós.

Aznar, A. y Castañón GonzÁlez, D. (2008). ¿Son o se hacen? El campo de la discapacidad intelectual estudiado a través de recorridos múltiples. Buenos Aires: Noveduc. 
Bonnin, M. (2007). La trama de las funciones museológicas. El caso del Museo de Antropología de Córdoba, Argentina. Tesis de maestría en Museología, Universidad Nacional, Costa Rica.

Chagas, M. (2007). La radiante aventura de los museos, en IX Seminario sobre Patrimonio Cultural. Santiago de Chile: Dirección de Bibliotecas, Archivos y Museos.

Díaz Morales, M. et al. (2018). La Psicopedagogía en el Museo: Discapacidad $y$ arte (inédito).

Edelstein, G. (1996). Un capítulo pendiente: El método en el debate didáctico contemporáneo, en A. Camilloni et al., Corrientes didácticas contemporáneas (pp. 75-90). Buenos Aires: Paidós.

Erausquin, C., Basualdo, M. E., Beraldo, V. y Aune S. (2010). Modelos Mentales en la intervención sobre situaciones-problema de la práctica docente en la Formación de Profesores en la Universidad: fortalezas y nudos críticos. En el marco del Proyecto UBACYT P023 "Los Psicólogos y la Fragmentación de la Experiencia Educativa: Modelos Mentales y Sistemas de Actividad para el Análisis y la Intervención en Problemas Situados en Contextos" de la Programación UBACYT 2008-2010.

Fernández, A. (2003). Los idiomas del Aprendiente. Buenos Aires: Ediciones Nueva Visión.

Giannone, A. y Gómez, M. (2011). La Psicopedagogía a la distancia. Desde 1930 hasta 1970 en Córdoba. Córdoba: Fundación Mannoni.

MendozA, M. (2016). Elementos para entender la extensión en la universidad. E+E: Estudios De Extensión En Humanidades 4(4), 29-36. Recuperado de: https://revistas.unc.edu.ar/index.php/EEH/article/view/15140

Muñoz-Padilla A. (2010). Discapacidad: conceptos, contextos y modelos. Pontificia Universidad Javeriana. Bogotá. Colombia. Recuperado el 6 de marzo de 2020 en https://www.redalyc.org/pdf/824/82420041012.pdf

NAVAJAs CorRal, Ó. (2008). Museos y museología: apuntes para una museología crítica. Versión ampliada de la ponencia presentada en el XXIX Congreso Anual del ICOFOM / XV Congreso Regional del ICOFOMA-LAM "Museología e historia: un campo de conocimiento".

Navajas Corral, Ó. (2008). Una "Nueva” Museología. ICOM Argentina / Universidad Antonio Nebrija. 
PACHeCo, M. Reflexiones en torno a la construcción del espacio de la extensión universitaria hoy (inédito).

Petz, I. (2015). Extensión universitaria: tendencias actuales y desafíos pendientes. Redes de extensión 1, 1-5.

Priego, C. (1989) Retratos y autorretratos en las miniaturas españolas altomedievales. Revista anual de Historia del arte 8, 7-34.

SAgÁstegui, D. (2004). Una apuesta por la cultura: el aprendizaje situado. Revista Electrónica Sinéctica 24, 30-39. México. Recuperado de: https:// www.redalyc.org/pdf/998/99815918005.pdf

ScheINER, T. (2018). Museología-poética, política y ética. Dimensiones transformadoras de las relaciones entre lo Humano y lo Real. ICOFOM Study Series 46. Recuperado de: https://journals.openedition.org/iss/1126

Wassermann, S. (1994) Introduction to case method teaching. Nueva York: Teachers College Press, Columbia University.

Zabala, M., Zabalza, P. y García Armesto, A. (2017). Educación en otros espacios. Reflexiones en torno a una propuesta de formación entre los ISFD de Córdoba y el Museo de Antropología (FFyH_UNC). Educación, Formación e Investigación. Revista de la Dirección general de Educación Superior 3(5). Córdoba: Ministerio de Educación. Recuperado de:_http://ppct.caicyt. gov.ar/index.php/efi/index

Zabala, M. y García Armesto, A. (2016). Construcción de un programa extensionista entre el Museo de Antropología y los institutos de formación docente: "Necesito observar una visita guiada". Revista Extensión en Red. Revista electrónica sobre extensión universitaria 7. FPyCS / Universidad Nacional de La Plata. Recuperado de: http://perio.unlp.edu.ar/ojs/index. php/extensionenred ISSN 1852-9569

\section{Documentos oficiales}

Reglamento del Museo de Antropología de la Facultad de Filosofía y Humanidades de la Universidad Nacional de Córdoba. Exp. 12/02/24545 Ord. N. ${ }^{\circ} 01 / 02$.

Ley Nacional de Educación n. ${ }^{\circ} 26.206$. 


\section{Agradecimientos}

Este trabajo fue posible gracias a la participación de los estudiantes de tercer y cuarto del nivel secundario de la Escuela Especial Doctora Carolina Ana Mosca junto a todos los directivos, docentes y auxiliares; a los practicantesestudiantes de la licenciatura en Psicopedagogía, cursando quinto año, de la Universidad Provincial, y a los trabajadores (guardias de seguridad, docentes, no docentes, estudiantes e investigadores) del Museo de Antropología de la Facultad de Filosofía y Humanidades de la Universidad Nacional de Córdoba.

El equipo de trabajo permanente estuvo conformado por las colegas de la Escuela Especial Dra. Carolina Ana Mosca, Mariana Dalmasso y Noel Arias; por la licenciatura de Psicopedagogía (UPC), Mariana Dalmasso y Valeria Andrea Durán; y por el Museo de Antropología (FFyH-UNC), Mariela Eleonora Zabala y Lucia Tamagnini.

A todos, nuestro agradecimiento. 